\title{
SPECTRAL DECOMPOSITION OF NORMAL OPERATOR IN REAL HILBERT SPACE
}

\author{
M.N. ORESHINA
}

\begin{abstract}
We consider normal unbounded operators acting in a real Hilbert space. The standard approach to solving spectral problems related with such operators is to apply the complexification, which is a passage to a complex space. At that, usually, the final results are to be decomplexified, that is, the reverse passage is needed. However, the decomplexification often turns out to be nontrivial.

The aim of the present paper is to extend the classical results of the spectral theory for the case of normal operators acting in a real Hilbert space. We provide two real versions of the spectral theorem for such operators.

We construct the functional calculus generated by the real spectral decomposition of a normal operator. We provide examples of using the obtained functional calculus for representing the exponent of a normal operator.
\end{abstract}

Keywords: unbounded normal operator, real Hilbert space, complexification, spectral theorem, functional calculus.

Mathematics Subject Classification: 47B15

\section{INTRODUCTION}

Many results in the theory of normal operators are based on the spectral theorem [1]-[5], which to each normal operator $N$ associates the resolution of the identity $E$ defined on Borel subsets $\mathbb{C}$ and supported on the spectrum of the operator $N$. By means of $E$, this allows one to represent the operator as some integral. At that one usually assumes that the operator $N$ acts in a complex Hilbert space and it is recommended to make the complexification in the real case. Nevertheless, in many applications, for instance, in numerics [6]-[14], it is desired to have statements formulated in terms of a real space.

In this paper we discuss the constructing of a real spectral decomposition in a real Hilbert space. In Section 2 we recall basic facts on unbounded normal operators and their complexification. To formulate the spectral theorem, in Section 3 we employ the representation of a normal operator as a sum of a self-adjoint and a skew-adjoint operator. As a result, the operator is expanded into the sum of two integrals and the spectral decomposition consists of two families of the operators defined on Borel sets in $\mathbb{R}$ and acting in a real Hilbert space. Despite such approach is natural, this turns out to be not very appropriate since we failed extending it to one of the most important results in the spectral theory, the construction of functional calculus. This is why in Section 4 we provide another version of the spectral theorem and in Section 5 we provide the corresponding theorem on functional calculus. In this case the spectral decomposition is again two families of the operators acting in a real Hilbert space but defined on Borel sets in the upper complex half-plane.

M.N. Oreshina,Spectral decomposition of a normal operator in a Real Hilbert space.

(C) ORESHINA M.N. 2017.

Submitted May 22, 2016. 


\section{COMPLEXIFICATION OF NORMAL OPERATOR}

Let $\mathbf{H}_{\mathbb{R}}$ be a real Hilbert space with a scalar product $\langle\cdot, \cdot\rangle_{\mathbb{R}}$.

Let $N_{\mathbb{R}}: D\left(N_{\mathbb{R}}\right) \subset \mathbf{H}_{\mathbb{R}} \rightarrow \mathbf{H}_{\mathbb{R}}$ be a linear unbounded operator. We assume the domain $D\left(N_{\mathbb{R}}\right)$ of the operator $N_{\mathbb{R}}$ is dense in $\mathbf{H}_{\mathbb{R}}$. The adjoint operator $N_{\mathbb{R}}^{*}$ is defined similar to the case of a complex Hilbert space [3]. An operator $A_{\mathbb{R}}$ is called self-adjoint if $A_{\mathbb{R}}=A_{\mathbb{R}}^{*}$. The operator $B_{\mathbb{R}}$ is called skew-adjoint if $B_{\mathbb{R}}=-B_{\mathbb{R}}^{*}$. An operator $N_{\mathbb{R}}$ is called normal if it is densely defined, is closed and satisfies the condition $N_{\mathbb{R}} N_{\mathbb{R}}^{*}=N_{\mathbb{R}}^{*} N_{\mathbb{R}}$. Similar to the complex case [3, Thm. 13.32] one can prove that a normal operator satisfies relation $D\left(N_{\mathbb{R}}\right)=D\left(N_{\mathbb{R}}^{*}\right)$. Self-adjoint and skew-adjoint operators are obviously normal.

Lemma 1. Let $B_{\mathbb{R}}: D\left(B_{\mathbb{R}}\right) \subset \mathbf{H}_{\mathbb{R}} \rightarrow \mathbf{H}_{\mathbb{R}}$ be a skew-adjoint operator. Then for each $\varphi \in$ $D\left(B_{\mathbb{R}}\right)$ the identity $\left\langle B_{\mathbb{R}} \varphi, \varphi\right\rangle_{\mathbb{R}}=0$ holds true.

The proof is reduced to straightforward calculations.

A linear space $\mathbf{H}_{\mathbb{R}} \times \mathbf{H}_{\mathbb{R}}$ over the field $\mathbb{C}$ with the rule of external multiplication by complex numbers $(\alpha+i \beta)(\varphi, \psi)=(\alpha \varphi-\beta \psi, \alpha \psi+\beta \varphi), \alpha, \beta \in \mathbb{R},(\varphi, \psi) \in \mathbf{H}_{\mathbb{R}} \times \mathbf{H}_{\mathbb{R}}$ is called complexification of the real Hilbert space $\mathbf{H}_{\mathbb{R}}$ and is denoted by $\mathbf{H}_{\mathbb{C}}$ [15], [16], [17], [18, [19]. It is convenient to write the elements in $\mathbf{H}_{\mathbb{C}}$ as $\varphi+i \psi$, where $\varphi, \psi \in \mathbf{H}_{\mathbb{R}}$ and $i$ is the imaginary unit. We shall identify $\mathbf{H}_{\mathbb{R}}$ with the subspace $\mathbf{H}_{\mathbb{R}} \times\{0\}$ of the space $\mathbf{H}_{\mathbb{C}}$. It is obvious that $\mathbf{H}_{\mathbb{C}}$ is a complex Hilbert space w.r.t. the scalar product

$$
\left\langle\varphi_{1}+i \psi_{1}, \varphi_{2}+i \psi_{2}\right\rangle_{\mathbb{C}}=\left\langle\varphi_{1}, \varphi_{2}\right\rangle_{\mathbb{R}}+\left\langle\psi_{1}, \psi_{2}\right\rangle_{\mathbb{R}}+i\left\langle\psi_{1}, \varphi_{2}\right\rangle_{\mathbb{R}}-i\left\langle\varphi_{1}, \psi_{2}\right\rangle_{\mathbb{R}} .
$$

The complexification of the operator $N_{\mathbb{R}}: D\left(N_{\mathbb{R}}\right) \subset \mathbf{H}_{\mathbb{R}} \rightarrow \mathbf{H}_{\mathbb{R}}$ is called [16]-[19] the operator $N_{\mathbb{C}}: D\left(N_{\mathbb{C}}\right) \subset \mathbf{H}_{\mathbb{C}} \rightarrow \mathbf{H}_{\mathbb{C}}$ with the domain $D\left(N_{\mathbb{C}}\right)=D\left(N_{\mathbb{R}}\right) \times D\left(N_{\mathbb{R}}\right)$ acting by the rule

$$
N_{\mathbb{C}}(\varphi+i \psi)=N_{\mathbb{R}} \varphi+i N_{\mathbb{R}} \psi, \quad \varphi, \psi \in D\left(N_{\mathbb{R}}\right) .
$$

Proposition 2. Let an operator $N_{\mathbb{C}}$ be the complexification of the operator $N_{\mathbb{R}}: D\left(N_{\mathbb{R}}\right) \subset$ $\mathbf{H}_{\mathbb{R}} \rightarrow \mathbf{H}_{\mathbb{R}}$, and $D\left(N_{\mathbb{R}}\right)$ is dense in $\mathbf{H}_{\mathbb{R}}$. Then

(a) the domain $D\left(N_{\mathbb{C}}\right)$ of the operator $N_{\mathbb{C}}$ is dense in $\mathbf{H}_{\mathbb{C}}$;

(b) the operator $N_{\mathbb{C}}{ }^{*}$ adjoint for $N_{\mathbb{C}}$ is the complexification of the adjoint operator $N_{\mathbb{R}}^{*}$;

(c) for a normal operator $N_{\mathbb{R}}$ the operator $N_{\mathbb{C}}$ is also normal.

The proposition can be checked by straightforward calculations.

By the symbols $\mathbf{0}_{\mathbb{C}}: \mathbf{H}_{\mathbb{C}} \rightarrow \mathbf{H}_{\mathbb{C}}$ and $\mathbf{0}_{\mathbb{R}}: \mathbf{H}_{\mathbb{R}} \rightarrow \mathbf{H}_{\mathbb{R}}$ we denote the zero operators, while $\mathbf{1}_{\mathbb{C}}: \mathbf{H}_{\mathbb{C}} \rightarrow \mathbf{H}_{\mathbb{C}}$ and $\mathbf{1}_{\mathbb{R}}: \mathbf{H}_{\mathbb{R}} \rightarrow \mathbf{H}_{\mathbb{R}}$ stand for the identity mappings. It is obvious that $\mathbf{0}_{\mathbb{C}}$ is a complexification of $\mathbf{0}_{\mathbb{R}}$ and $\mathbf{1}_{\mathbb{C}}$ is the complexification of $\mathbf{1}_{\mathbb{R}}$.

The inverse operator of an unbounded operator $N_{\mathbb{R}}: D\left(N_{\mathbb{R}}\right) \subset \mathbf{H}_{\mathbb{R}} \rightarrow \mathbf{H}_{\mathbb{R}}$ is the operator $N_{\mathbb{R}}^{-1}: \mathbf{H}_{\mathbb{R}} \rightarrow D\left(N_{\mathbb{R}}\right)$ satisfying the identities

$$
N_{\mathbb{R}} N_{\mathbb{R}}^{-1} \varphi=\varphi, \quad \varphi \in \mathbf{H}_{\mathbb{R}} ; \quad N_{\mathbb{R}}^{-1} N_{\mathbb{R}} \psi=\psi, \quad \psi \in D\left(N_{\mathbb{R}}\right) .
$$

Let $N_{\mathbb{C}}$ be a closed operator. The set $\rho\left(N_{\mathbb{C}}\right)$ of all $\lambda \in \mathbb{C}$, for which the operator $\lambda \mathbf{1}_{\mathbb{C}}-N_{\mathbb{C}}$ has a bounded inverse, is called the resolvent set [3]-[5] of the operator $N_{\mathbb{C}}$, while the function $\lambda \mapsto\left(\lambda \mathbf{1}_{\mathbb{C}}-N_{\mathbb{C}}\right)^{-1}$ is called the resolvent. The complement $\sigma\left(N_{\mathbb{C}}\right)$ of the resolvent set is called the spectrum of the operator $N_{\mathbb{C}}$. The spectrum of a closed operator is a closed set [3], [5]. It can be shown [3] that the spectrum of the self-adjoint operator is located on the real axis. For a normal operator $N_{\mathbb{R}}$ acting in the space $\mathbf{H}_{\mathbb{R}}$, we shall make use of the following auxiliary sets: the projections of the spectrum of its complexification $N_{\mathbb{C}}$ on the real and the imaginary axes

$$
\sigma^{\operatorname{Re}}\left(N_{\mathbb{R}}\right)=\sigma^{\operatorname{Re}}\left(N_{\mathbb{C}}\right)=\left\{\operatorname{Re} \lambda: \lambda \in \sigma\left(N_{\mathbb{C}}\right)\right\}, \quad \sigma^{\operatorname{Im}}\left(N_{\mathbb{R}}\right)=\sigma^{\operatorname{Im}}\left(N_{\mathbb{C}}\right)=\left\{\operatorname{Im} \lambda: \lambda \in \sigma\left(N_{\mathbb{C}}\right)\right\} ;
$$

the projection of the spectrum of the complexification $N_{\mathbb{C}}$ on the non-negative imaginary axis

$$
\sigma^{\operatorname{Im}+}\left(N_{\mathbb{R}}\right)=\sigma^{\operatorname{Im}+}\left(N_{\mathbb{C}}\right)=\left\{\operatorname{Im} \lambda: \lambda \in \sigma\left(N_{\mathbb{C}}\right), \operatorname{Im} \lambda \geqslant 0\right\},
$$


the pure real spectrum

$$
\sigma^{0}\left(N_{\mathbb{R}}\right)=\sigma^{0}\left(N_{\mathbb{C}}\right)=\left\{\lambda \in \sigma\left(N_{\mathbb{C}}\right): \operatorname{Im} \lambda=0\right\},
$$

and the upper part (w.r.t. the real axis) of the spectrum of the complexification $N_{\mathbb{C}}$ :

$$
\sigma^{+}\left(N_{\mathbb{R}}\right)=\sigma^{+}\left(N_{\mathbb{C}}\right)=\left\{\lambda \in \sigma\left(N_{\mathbb{C}}\right): \operatorname{Im} \lambda \geqslant 0\right\} .
$$

For $\omega \subset \mathbb{C}$, by $\bar{\omega} \subset \mathbb{C}$ we denote the set $\{\bar{\xi}: \xi \in \omega\}$ consisting of adjoint numbers. Thus, the set $\bar{\omega}$ is symmetric with the set $\omega$ w.r.t. the real axis. We note that the notation $\bar{\omega}$ is often used to denote the closure of a set $\omega$, but in the present paper the closure of a set $\omega$ is denoted by $[\omega]$.

By $J: \mathbf{H}_{\mathbb{C}} \rightarrow \mathbf{H}_{\mathbb{C}}$ we denote the operator defined by the formula

$$
J(\varphi+i \psi)=\varphi-i \psi, \quad \varphi, \psi \in \mathbf{H}_{\mathbb{R}} .
$$

It is obvious that $J^{2}=\mathbf{1}_{\mathbb{C}}$ and $J^{-1}=J$. We note that the operator $J$ is adjoint-linear, that is, $J(\varphi+\psi)=J \varphi+J \psi, J(\xi \varphi)=\bar{\xi} J \varphi$ for all $\varphi, \psi \in \mathbf{H}_{\mathbb{C}}, \xi \in \mathbb{C}$.

We provide a criterion for the possibility to decomplexify an operator $N_{\mathbb{C}}$, that is, to represent it as the complexification of an operator acting in $\mathbf{H}_{\mathbb{R}}$. A similar statement was provided in [16, Lm. 3.5].

Lemma 3. The operator $N_{\mathbb{C}}: D\left(N_{\mathbb{C}}\right) \subset \mathbf{H}_{\mathbb{C}} \rightarrow \mathbf{H}_{\mathbb{C}}$ is the complexification of some operator $N_{\mathbb{R}}: D\left(N_{\mathbb{R}}\right) \subset \mathbf{H}_{\mathbb{R}} \rightarrow \mathbf{H}_{\mathbb{R}}$ if and only if it commutes with the operator $J$ or, equivalently, the relation $J N_{\mathbb{C}} J=N_{\mathbb{C}}$ holds true.

The lemma is checked by straightforward calculations.

The next proposition states that the spectrum and the resolvent set of the complexification are symmetric w.r.t. the real axis.

Proposition 4. Let $N_{\mathbb{C}}: D\left(N_{\mathbb{C}}\right) \subset \mathbf{H}_{\mathbb{C}} \rightarrow \mathbf{H}_{\mathbb{C}}$ be the complexification of an operator $N_{\mathbb{R}}: D\left(N_{\mathbb{R}}\right) \subset \mathbf{H}_{\mathbb{R}} \rightarrow \mathbf{H}_{\mathbb{R}}$. Then $\overline{\rho\left(N_{\mathbb{C}}\right)}=\rho\left(N_{\mathbb{C}}\right)$ and $\overline{\sigma\left(N_{\mathbb{C}}\right)}=\sigma\left(N_{\mathbb{C}}\right)$.

The proof reproduces that of Lemma 4.1 in [16].

\section{FIRST SPECTRAL THEOREM (ON REAL AXIS)}

By $\mathcal{B}\left(\mathbf{H}_{\mathbb{C}}\right)$ we denote the Banach algebra [2]-4] of all linear bounded operators acting in $\mathbf{H}_{\mathbb{C}}$. In the same way we define $\mathcal{B}\left(\mathbf{H}_{\mathbb{R}}\right)$. An operator $P \in \mathcal{B}\left(\mathbf{H}_{\mathbb{C}}\right)\left(\right.$ or $P \in \mathcal{B}\left(\mathbf{H}_{\mathbb{R}}\right)$ ) is called a projector if $P^{2}=P$. An operator $P \in \mathcal{B}\left(\mathbf{H}_{\mathbb{C}}\right)$ is called self-adjoint if $\langle P \varphi, \psi\rangle_{\mathbb{C}}=\langle\varphi, P \psi\rangle_{\mathbb{C}}, \varphi, \psi \in \mathbf{H}_{\mathbb{C}}$. An operator $P \in \mathcal{B}\left(\mathbf{H}_{\mathbb{C}}\right)$ is called skew-adjoint if $\langle P \varphi, \psi\rangle_{\mathbb{C}}=-\langle\varphi, P \psi\rangle_{\mathbb{C}}, \varphi, \psi \in \mathbf{H}_{\mathbb{C}}$. In the same way we define a self-adjoint and a skew-adjoint operator in $\mathcal{B}\left(\mathbf{H}_{\mathbb{R}}\right)$. We note that these definitions can be regarded as the particular cases of the definition of unbounded self-adjoint and skew-adjoint operators in the previous section. Lemma 1 is obviously true for a bounded skew-adjoint operator.

Let $\Omega$ be a Borel subset of $\mathbb{R}$ or $\mathbb{C}$. The (complex) resolution of the identity [3] on the $\sigma$-algebra $\Sigma$ of all Borel subsets of $\mathbb{R}$ or $\mathbb{C}$ supported by a set $\Omega$ is the mapping $E: \Sigma \rightarrow \mathcal{B}\left(\mathbf{H}_{\mathbb{C}}\right)$ possessing the properties:

1) $E(\mathbb{R} \backslash \Omega)=\mathbf{0}_{\mathbb{C}},\left(E(\mathbb{C} \backslash \Omega)=\mathbf{0}_{\mathbb{C}}\right), E(\Omega)=\mathbf{1}_{\mathbb{C}}$;

2) for each $\omega \in \Sigma$, the operator $E(\omega)$ is a self-adjoint projector;

3) the identity $E\left(\omega^{\prime} \cap \omega^{\prime \prime}\right)=E\left(\omega^{\prime}\right) E\left(\omega^{\prime \prime}\right)$ holds for all $\omega^{\prime}, \omega^{\prime \prime} \in \Sigma$;

4) the identity $E\left(\omega^{\prime} \cup \omega^{\prime \prime}\right)=E\left(\omega^{\prime}\right)+E\left(\omega^{\prime \prime}\right)$ holds for all $\omega^{\prime}, \omega^{\prime \prime} \in \Sigma, \omega^{\prime} \cap \omega^{\prime \prime}=\varnothing$;

5) for all $\varphi, \psi \in \mathbf{H}_{\mathbb{C}}$ the function $E_{\varphi \psi}(\omega)=\langle E(\omega) \varphi, \psi\rangle_{\mathbb{C}}$ is a complex measure [3], [20], 21] on $\Sigma$. 
Let us recall the spectral theorem for a normal operator $N_{\mathbb{C}}$ acting in the complex Hilbert space. The complex resolution of the identity discussed in this theorem is called the (complex) spectral decomposition of an operator $N_{\mathbb{C}}$. It is denoted by $E^{N_{\mathbb{C}}}$ to stress that it is related with the operator $N_{\mathbb{C}}$.

Theorem 5 ([3, Thm. 13.33]). Let $N_{\mathbb{C}}: D\left(N_{\mathbb{C}}\right) \subset \mathbf{H}_{\mathbb{C}} \rightarrow \mathbf{H}_{\mathbb{C}}$ be a normal operator. Then there exists the unique complex resolution of the identity $E^{N_{\mathbb{C}}}$ supported by $\sigma\left(N_{\mathbb{C}}\right) \subset \mathbb{C}$ satisfying the relation

$$
\left\langle N_{\mathbb{C}} \varphi, \psi\right\rangle_{\mathbb{C}}=\int_{\mathbb{C}} \xi d E_{\varphi \psi}^{N_{\mathbb{C}}}(\xi), \quad \varphi \in D\left(N_{\mathbb{C}}\right), \quad \psi \in \mathbf{H}_{\mathbb{C}}
$$

Moreover, $E^{N_{\mathbb{C}}}(\omega) S=S E^{N_{\mathbb{C}}}(\omega)$ for each set $\omega \subset \Sigma$ and each operator $S \in \mathcal{B}\left(\mathbf{H}_{\mathbb{C}}\right)$ commuting with the operator $N_{\mathbb{C}}$ in the sense that $S N_{\mathbb{C}} \subseteq N_{\mathbb{C}} S$.

Assume that we are given the spectral decomposition $E^{N_{\mathbb{C}}}$ of a normal operator $N_{\mathbb{C}}$. The essential range of a Borel measurable function $f: \mathbb{C} \rightarrow \mathbb{C}$ is the intersection of the sets of form $[f(\omega)]$ for all $\omega \in \Sigma$ such that $E^{N_{\mathbb{C}}}(\omega)=\mathbf{1}_{\mathbb{C}}$, where [.] denotes the closure of a set [3], [5]. The function $f$ is called essentially bounded w.r.t. $E^{N_{\mathbb{C}}}$ if its essential range is bounded [3], [5].

In a complex Hilbert space, the following theorem on functional calculus is true.

Theorem 6 ([3], Thm. 13.24, 13.25, 13.27]). Let $E^{N_{\mathbb{C}}}$ be the spectral decomposition of a normal operator $N_{\mathbb{C}}: D\left(N_{\mathbb{C}}\right) \subset \mathbf{H}_{\mathbb{C}} \rightarrow \mathbf{H}_{\mathbb{C}}$. To each Borel measurable function $f: \mathbb{C} \rightarrow \mathbb{C}$, the formula

$$
\left\langle\Psi_{\mathbb{C}}(f) \varphi, \psi\right\rangle_{\mathbb{C}}=\int_{\mathbb{C}} f(\xi) d E_{\varphi \psi}^{N_{\mathbb{C}}}(\xi), \quad \varphi \in D\left(\Psi_{\mathbb{C}}(f)\right), \quad \psi \in \mathbf{H}_{\mathbb{C}}
$$

associates a densely defined closed operator

$$
\Psi_{\mathbb{C}}(f): D\left(\Psi_{\mathbb{C}}(f)\right) \subset \mathbf{H}_{\mathbb{C}} \rightarrow \mathbf{H}_{\mathbb{C}}
$$

with the domain $D\left(\Psi_{\mathbb{C}}(f)\right)=\left\{\varphi \in \mathbf{H}_{\mathbb{C}}: \int_{\mathbb{C}}|f|^{2} d E_{\varphi \varphi}^{N_{\mathbb{C}}}<\infty\right\}$. At that, the mapping $\Psi_{\mathbb{C}}$ possesses the following properties:

(a) For each $\varphi \in D\left(\Psi_{\mathbb{C}}(f)\right)$ the relation

$$
\left\|\Psi_{\mathbb{C}}(f) \varphi\right\|_{\mathbb{C}}^{2}=\int_{\mathbb{C}}|f|^{2} d E_{\varphi \varphi}^{N_{\mathbb{C}}}
$$

holds true.

(b) The operator $\Psi_{\mathbb{C}}(f)$ is normal and the identities

$$
\Psi_{\mathbb{C}}(f)^{*}=\Psi_{\mathbb{C}}(\bar{f}), \quad \Psi_{\mathbb{C}}(f) \Psi_{\mathbb{C}}(f)^{*} \quad=\Psi_{\mathbb{C}}\left(|f|^{2}\right)=\Psi_{\mathbb{C}}(f)^{*} \Psi_{\mathbb{C}}(f)
$$

hold true.

(c) If a function $f: \mathbb{C} \rightarrow \mathbb{C}$ is essentially bounded w.r.t. $E^{N_{\mathbb{C}}}$, then the operator $\Psi_{\mathbb{C}}(f)$ is bounded.

(d) For all Borel measurable functions $f, g: \mathbb{C} \rightarrow \mathbb{C}$ the inclusions

$$
\Psi_{\mathbb{C}}(f)+\Psi_{\mathbb{C}}(g) \subseteq \Psi_{\mathbb{C}}(f+g), \quad \Psi_{\mathbb{C}}(f) \Psi_{\mathbb{C}}(g) \subseteq \Psi_{\mathbb{C}}(f g)
$$

hold. If at that the function $g$ is bounded, then

$$
\Psi_{\mathbb{C}}(f)+\Psi_{\mathbb{C}}(g)=\Psi_{\mathbb{C}}(f+g), \quad \Psi_{\mathbb{C}}(f) \Psi_{\mathbb{C}}(g)=\Psi_{\mathbb{C}}(f g) .
$$

(e) The spectrum $\sigma\left(\Psi_{\mathbb{C}}(f)\right)$ of the operator $\Psi_{\mathbb{C}}(f)$ is the essential range of the function $f$.

The formula (2) is often shortly written as $\Psi_{\mathbb{C}}(f)=\int_{\mathbb{C}} f d E^{N_{\mathbb{C}}}$.

The next theorem is called the change of measure principle [3]. 
Theorem 7. Let $E$ be a complex resolution of the identity on a $\sigma$-algebra $\Sigma$ supported on a set $\Omega$ and $\Omega^{\prime} \in \Sigma$. Assume that we are given a mapping $g: \Omega \rightarrow \Omega^{\prime}$, for which $\omega^{\prime} \in \Sigma$ implies $g^{-1}\left(\omega^{\prime}\right) \in \Sigma$. Then the mapping $E^{\prime}: \Sigma \rightarrow \mathcal{B}\left(\mathbf{H}_{\mathbb{C}}\right)$ defined by the formula $E^{\prime}\left(\omega^{\prime}\right)=E\left(g^{-1}(\omega)\right)$ is the complex resolution of the identity supported on $\Omega^{\prime}$ and at that,

$$
\int_{\mathbb{C}} f d E_{\varphi \psi}^{\prime}=\int_{\mathbb{C}} f \circ g d E_{\varphi \psi}
$$

for each Borel measurable function $f: \Omega^{\prime} \rightarrow \mathbb{C}$ and for each $\varphi$ and $\psi$, for which at least one of the integrals exists.

The proof reproduces that of Theorem 13.28 in [3].

Corollary 8. If under the assumptions of Theorem 7 the inclusion $\Omega^{\prime} \subset \mathbb{R}$ holds, then

$$
\int_{-\infty}^{+\infty} f d E_{\varphi \psi}^{\prime}=\int_{\mathbb{C}} f \circ g d E_{\varphi \psi}
$$

This corollary is implied immediately by Theorem 7 .

Proposition 9. Let $N_{\mathbb{C}}: D\left(N_{\mathbb{C}}\right) \subset \mathbf{H}_{\mathbb{C}} \rightarrow \mathbf{H}_{\mathbb{C}}$ be the complexification of a normal operator $N_{\mathbb{R}}: D\left(N_{\mathbb{R}}\right) \subset \mathbf{H}_{\mathbb{R}} \rightarrow \mathbf{H}_{\mathbb{R}}$. Then for the operators $E^{N_{\mathbb{C}}}(\omega)$ in the spectral decomposition of the operator $N_{\mathbb{C}}$ the relation

holds true.

$$
J E^{N_{\mathbb{C}}}(\omega) J=E^{N_{\mathbb{C}}}(\bar{\omega}), \quad \omega \in \Sigma,
$$

This proposition is implied by Proposition 2, Lemma 3, Theorem 5 and Theorem 6(b).

Corollary 10. Let $A_{\mathbb{C}}: D\left(A_{\mathbb{C}}\right) \subset \mathbf{H}_{\mathbb{C}} \rightarrow \mathbf{H}_{\mathbb{C}}$ be the complexification of a self-adjoint operator $A_{\mathbb{R}}: D\left(A_{\mathbb{R}}\right) \subset \mathbf{H}_{\mathbb{R}} \rightarrow \mathbf{H}_{\mathbb{R}}$. Then the operators $E^{A_{\mathbb{C}}}(\omega), \omega \in \Sigma$, in the spectral decomposition of the operator $A_{\mathbb{C}}$ commutes with the operator $J$ and therefore, they are the complexifications of some operators.

Proof. Since $E^{A_{\mathbb{C}}}$ is supported on $\sigma\left(A_{\mathbb{C}}\right) \subset \mathbb{R}$, then $J E^{A_{\mathbb{C}}}(\omega) J=E^{A_{\mathbb{C}}}(\bar{\omega})=E^{A_{\mathbb{C}}}(\omega)$.

Theorem 11. Let $N_{\mathbb{C}}: D\left(N_{\mathbb{C}}\right) \subset \mathbf{H}_{\mathbb{C}} \rightarrow \mathbf{H}_{\mathbb{C}}$ be the complexification of a normal operator $N_{\mathbb{R}}: D\left(N_{\mathbb{R}}\right) \subset \mathbf{H}_{\mathbb{R}} \rightarrow \mathbf{H}_{\mathbb{R}}$, and $f: \mathbb{C} \rightarrow \mathbb{C}$ is a Borel measurable function. Then the operator $\Psi_{\mathbb{C}}(f)$ possesses the property $J \Psi_{\mathbb{C}}(f) J=\Psi_{\mathbb{C}}(\widetilde{f})$, where $\widetilde{f}(\xi)=\overline{f(\bar{\xi})}$.

The theorem can be checked by straightforward calculations.

Corollary 12. Let $N_{\mathbb{C}}: D\left(N_{\mathbb{C}}\right) \subset \mathbf{H}_{\mathbb{C}} \rightarrow \mathbf{H}_{\mathbb{C}}$ be the complexification of a normal operator $N_{\mathbb{R}}: D\left(N_{\mathbb{R}}\right) \subset \mathbf{H}_{\mathbb{R}} \rightarrow \mathbf{H}_{\mathbb{R}}$, and $f: \mathbb{C} \rightarrow \mathbb{C}$ is a Borel measurable function possessing the property $f(\xi)=\overline{f(\bar{\xi})}$. Then the operator $\Psi_{\mathbb{C}}(f)$ commutes with the operator $J$ and therefore, it is a complexification of some operator.

This corollary is implied by Theorem 11

Proposition 13. Let $N_{\mathbb{C}}: D\left(N_{\mathbb{C}}\right) \subset \mathbf{H}_{\mathbb{C}} \rightarrow \mathbf{H}_{\mathbb{C}}$ be a normal operator. Then there exists a self-adjoint operator $A_{\mathbb{C}}: D\left(A_{\mathbb{C}}\right) \subset \mathbf{H}_{\mathbb{C}} \rightarrow \mathbf{H}_{\mathbb{C}}$ and a skew-adjoint operator $B_{\mathbb{C}}: D\left(B_{\mathbb{C}}\right) \subset$ $\mathbf{H}_{\mathbb{C}} \rightarrow \mathbf{H}_{\mathbb{C}}$ such that

(a) the representations $N_{\mathbb{C}}=A_{\mathbb{C}}+B_{\mathbb{C}}, N_{\mathbb{C}}^{*}=A_{\mathbb{C}}-B_{\mathbb{C}}$ hold true;

(b) their spectra satisfy the formulae $\sigma\left(A_{\mathbb{C}}\right)=\sigma^{\operatorname{Re}}\left(N_{\mathbb{C}}\right), \sigma\left(B_{\mathbb{C}}\right)=i \sigma^{\operatorname{Im}}\left(N_{\mathbb{C}}\right)$;

(c) the operators $A_{\mathbb{C}}$ and $B_{\mathbb{C}}$ commutes on $D\left(N_{\mathbb{C}} N_{\mathbb{C}}{ }^{*}\right)$;

(d) if $N_{\mathbb{C}}$ is the complexification of a normal operator $N_{\mathbb{R}}: D\left(N_{\mathbb{R}}\right) \subset \mathbf{H}_{\mathbb{R}} \rightarrow \mathbf{H}_{\mathbb{R}}$, then the operators $A_{\mathbb{C}}$ and $B_{\mathbb{C}}$ commute with the operator $J$ and, therefore, are the complexifications of some operators. 
Proof. We introduce the auxiliary functions $f(\xi)=\operatorname{Re} \xi, g(\xi)=i \operatorname{Im} \xi$, and according Theorem 6 we let

$$
\begin{aligned}
& A_{\mathbb{C}}=\Psi_{\mathbb{C}}(f)=\int_{\mathbb{C}} \operatorname{Re} \xi d E^{N_{\mathbb{C}}}(\xi), \\
& D\left(A_{\mathbb{C}}\right)=\left\{\varphi \in \mathbf{H}_{\mathbb{C}}: \int_{\mathbb{C}}(\operatorname{Re} \xi)^{2} d E_{\varphi \varphi}^{N_{\mathbb{C}}}(\xi)<\infty\right\}, \\
& B_{\mathbb{C}}=\Psi_{\mathbb{C}}(g)=\int_{\mathbb{C}} i \operatorname{Im} \xi d E^{N_{\mathbb{C}}}(\xi), \\
& D\left(B_{\mathbb{C}}\right)=\left\{\varphi \in \mathbf{H}_{\mathbb{C}}: \int_{\mathbb{C}}(\operatorname{Im} \xi)^{2} d E_{\varphi \varphi}^{N_{\mathbb{C}}}(\xi)<\infty\right\} .
\end{aligned}
$$

At that, the following identities are obvious: $N_{\mathbb{C}}=A_{\mathbb{C}}+B_{\mathbb{C}}, N_{\mathbb{C}}{ }^{*}=A_{\mathbb{C}}-B_{\mathbb{C}}$.

Other statements of this theorem can be checked by straightforward calculations.

Corollary 14. Let $N_{\mathbb{R}}: D\left(N_{\mathbb{R}}\right) \subset \mathbf{H}_{\mathbb{R}} \rightarrow \mathbf{H}_{\mathbb{R}}$ be a normal operator. Then there exist a selfadjoint operator $A_{\mathbb{R}}: D\left(A_{\mathbb{R}}\right) \subset \mathbf{H}_{\mathbb{R}} \rightarrow \mathbf{H}_{\mathbb{R}}$ and a skew-adjoint operator $B_{\mathbb{R}}: D\left(B_{\mathbb{R}}\right) \subset \mathbf{H}_{\mathbb{R}} \rightarrow$ $\mathbf{H}_{\mathbb{R}}$ such that the representations $N_{\mathbb{R}}=A_{\mathbb{R}}+B_{\mathbb{R}}, N_{\mathbb{R}}^{*}=A_{\mathbb{R}}-B_{\mathbb{R}}$ hold. The operators $A_{\mathbb{R}}$ and $B_{\mathbb{R}}$ commute on $D\left(N_{\mathbb{R}} N_{\mathbb{R}}^{*}\right)$.

The corollary is implied by Proposition 13 .

Let $\Omega$ be Borel subset of $\mathbb{R}$ or $\mathbb{C}$. The real resolution of the identity on the $\sigma$-algebra $\Sigma$ of all Borel subsets of $\mathbb{R}$ or $\mathbb{C}$ supported on the set $\Omega$ is the mapping $E: \Sigma \rightarrow \mathcal{B}\left(\mathbf{H}_{\mathbb{R}}\right)$ possessing the properties:

1) $E(\mathbb{R} \backslash \Omega)=\mathbf{0}_{\mathbb{R}},\left(E(\mathbb{C} \backslash \Omega)=\mathbf{0}_{\mathbb{R}}\right), E(\Omega)=\mathbf{1}_{\mathbb{R}}$;

2) for each $\omega \in \Sigma$, the operator $E(\omega)$ is a self-adjoint projector;

3) for all $\omega^{\prime}, \omega^{\prime \prime} \in \Sigma$ the identity $E\left(\omega^{\prime} \cap \omega^{\prime \prime}\right)=E\left(\omega^{\prime}\right) E\left(\omega^{\prime \prime}\right)$ holds;

4) for all $\omega^{\prime}, \omega^{\prime \prime} \in \Sigma, \omega^{\prime} \cap \omega^{\prime \prime}=\varnothing$, the identity $E\left(\omega^{\prime} \cup \omega^{\prime \prime}\right)=E\left(\omega^{\prime}\right)+E\left(\omega^{\prime \prime}\right)$ holds;

5) for each $\varphi, \psi \in \mathbf{H}_{\mathbb{R}}$ the function $E_{\varphi \psi}(\omega)=\langle E(\omega) \varphi, \psi\rangle_{\mathbb{R}}$ is a real measure on $\Sigma$.

It follows from properties 1 and 3 that $E(\varnothing)=\mathbf{0}_{\mathbb{R}}$ and $E(\omega \cap \Omega)=E(\omega)$. The property 3 implies also that each two projectors $E(\omega)$ and $E\left(\omega^{\prime}\right)$ commute. The property 2 implies that for all $\varphi \in \mathbf{H}_{\mathbb{R}}$ the measure $E_{\varphi \varphi}(\omega)=\langle E(\omega) \varphi, \varphi\rangle_{\mathbb{R}}$ is positive.

Let $P_{\mathbb{R}} \in \mathcal{B}\left(\mathbf{H}_{\mathbb{R}}\right)$ be a self-adjoint projector. It is obvious that the operator $\mathbf{1}_{\mathbb{R}}-P_{\mathbb{R}}$ is also a self-adjoint projector. By $\mathcal{R}\left(\mathbf{1}_{\mathbb{R}}-P_{\mathbb{R}}\right)$ we denote the image of the operator $\mathbf{1}_{\mathbb{R}}-P_{\mathbb{R}}$. Let $\Delta$ be a Borel subset in the non-negative real axis $\mathbb{R}^{+}=[0,+\infty)$ or in the upper complex half-plane $\mathbb{C}^{+}=\{\lambda \in \mathbb{C}: \operatorname{Im} \lambda \geqslant 0\}$. The real skew resolution of the identity in the subspace $\mathcal{R}\left(\mathbf{1}_{\mathbb{R}}-P_{\mathbb{R}}\right)$ on the $\sigma$-algebra $\Sigma$ of all Borel subsets $\mathbb{R}^{+}$or $\mathbb{C}^{+}$supported on the set $\Delta$ is the mapping $G: \Sigma \rightarrow \mathcal{B}\left(\mathbf{H}_{\mathbb{R}}\right)$ possessing the properties

1) $G\left(\mathbb{R}^{+} \backslash \Delta\right)=\mathbf{0}_{\mathbb{R}}\left(G\left(\mathbb{C}^{+} \backslash \Delta\right)=\mathbf{0}_{\mathbb{R}}\right),(G(\Delta))^{2}=-\mathbf{1}_{\mathbb{R}}+P_{\mathbb{R}}$;

2) for each $\omega \in \Sigma$, the operator $G(\omega)$ is skew-adjoint;

3) for all $\omega^{\prime}, \omega^{\prime \prime}, \omega^{\prime \prime \prime} \in \Sigma$ the identity $G\left(\omega^{\prime} \cap \omega^{\prime \prime} \cap \omega^{\prime \prime \prime}\right)=-G\left(\omega^{\prime}\right) G\left(\omega^{\prime \prime}\right) G\left(\omega^{\prime \prime \prime}\right)$ holds;

4) for each $\omega^{\prime}, \omega^{\prime \prime} \in \Sigma, \omega^{\prime} \cap \omega^{\prime \prime}=\varnothing$, the identity $G\left(\omega^{\prime} \cup \omega^{\prime \prime}\right)=G\left(\omega^{\prime}\right)+G\left(\omega^{\prime \prime}\right)$ holds;

5) for all $\varphi, \psi \in \mathbf{H}_{\mathbb{R}}$ the function $G_{\varphi \psi}(\omega)=\langle G(\omega) \varphi, \psi\rangle_{\mathbb{R}}$ is a real measure on $\Sigma$.

Properties 1 and 3 imply that $G(\varnothing)=\mathbf{0}_{\mathbb{R}}$ and $G(\omega \cap \Delta)=G(\omega)\left(\mathbf{1}_{\mathbb{R}}-P_{\mathbb{R}}\right)=\left(\mathbf{1}_{\mathbb{R}}-P_{\mathbb{R}}\right) G(\omega)$. Property 3 implies also that $(G(\omega))^{3}=-G(\omega)$. Lemma 1 and Property 2 yield that $G_{\varphi \varphi}(\omega)=$ $\langle G(\omega) \varphi, \varphi\rangle_{\mathbb{R}}=0$.

In the same way we define the complex skew resolution of the identity in the subspace $\mathcal{R}\left(\mathbf{1}_{\mathbb{C}}-P_{\mathbb{C}}\right)$ corresponding to the projector $P_{\mathbb{C}} \in \mathcal{B}\left(\mathbf{H}_{\mathbb{C}}\right)$.

Theorem 15. Let $N_{\mathbb{R}}: D\left(N_{\mathbb{R}}\right) \subset \mathbf{H}_{\mathbb{R}} \rightarrow \mathbf{H}_{\mathbb{R}}$ be a normal operator. Then there exists a real resolution of the identity $E^{A_{\mathbb{R}}}$ supported on $\sigma^{\operatorname{Re}}\left(N_{\mathbb{R}}\right) \subset \mathbb{R}$, the self-adjoint projector $P_{\mathbb{R}}$ 
and the real skew resolution of the identity $G^{B_{\mathbb{R}}}$ in the subspace $\mathcal{R}\left(\mathbf{1}_{\mathbb{R}}-P_{\mathbb{R}}\right)$ supported on $\sigma^{\operatorname{Im}}+\left(N_{\mathbb{R}}\right) \subset \mathbb{R}^{+}$, for which the relation

$$
\left\langle N_{\mathbb{R}} \varphi, \psi\right\rangle_{\mathbb{R}}=\int_{-\infty}^{+\infty} \alpha d E_{\varphi \psi}^{A_{\mathbb{R}}}(\alpha)+\int_{0}^{+\infty} \beta d G_{\varphi \psi}^{B_{\mathbb{R}}}(\beta), \quad \varphi \in D\left(N_{\mathbb{R}}\right), \quad \psi \in \mathbf{H}_{\mathbb{R}},
$$

holds. At that, each two operators $E^{A_{\mathbb{R}}}(\omega)$ and $G^{B_{\mathbb{R}}}\left(\omega^{\prime}\right)$ commute.

The complexification $P_{\mathbb{C}}$ of the projector $P_{\mathbb{R}}$ and the complexifications $E^{A_{\mathbb{C}}}(\omega), G^{B_{\mathbb{C}}}(\omega)$ of the operators $E^{A_{\mathbb{R}}}(\omega), G^{B_{\mathbb{R}}}(\omega)$ are related with the spectral decomposition $E^{N_{\mathbb{C}}}$ of the complexification $N_{\mathbb{C}}$ of the operator $N_{\mathbb{R}}$ by the formulae

$$
\begin{aligned}
P_{\mathbb{C}} & =E^{N_{\mathbb{C}}}\left(\sigma^{0}\left(N_{\mathbb{R}}\right)\right), \\
E^{A_{\mathbb{C}}}(\omega) & =E^{N_{\mathbb{C}}}(\omega+i \mathbb{R}), \\
G^{B_{\mathbb{C}}}(\omega) & =i E^{N_{\mathbb{C}}}(\mathbb{R}+i \omega)-i E^{N_{\mathbb{C}}}(\mathbb{R}-i \omega),
\end{aligned}
$$

where $\omega+i \mathbb{R}=\{\alpha+i \beta: \alpha \in \omega, \beta \in \mathbb{R}\}, \mathbb{R} \pm i \omega=\{\alpha \pm i \beta: \alpha \in \mathbb{R}, \beta \in \omega\}$.

Proof. Let $N_{\mathbb{C}}$ be the complexification of an operator $N_{\mathbb{R}}$. According Proposition 13 and Corollary 14, we represent the operators $N_{\mathbb{C}}$ and $N_{\mathbb{R}}$ as $N_{\mathbb{C}}=A_{\mathbb{C}}+B_{\mathbb{C}}, N_{\mathbb{R}}=A_{\mathbb{R}}+B_{\mathbb{R}}$, where $A_{\mathbb{C}}$ and $A_{\mathbb{R}}$ are self-adjoint operators and $B_{\mathbb{C}}$ and $B_{\mathbb{R}}$ are skew-adjoint. It follows from (3) that for the operator $A_{\mathbb{C}}$ the representation

$$
\left\langle A_{\mathbb{C}} \varphi, \psi\right\rangle_{\mathbb{C}}=\int_{\mathbb{C}} \operatorname{Re} \xi d E_{\varphi \psi}^{N_{\mathbb{C}}}(\xi), \quad \varphi \in D\left(A_{\mathbb{C}}\right), \quad \psi \in \mathbf{H}_{\mathbb{C}}
$$

holds true. On the other hand, for the self-adjoint operator $A_{\mathbb{C}}$ there exists the resolution of the identity $E^{A_{\mathbb{C}}}$ and the representation holds:

$$
\left\langle A_{\mathbb{C}} \varphi, \psi\right\rangle_{\mathbb{C}}=\int_{-\infty}^{+\infty} \alpha d E_{\varphi \psi}^{A_{\mathbb{C}}}(\alpha), \quad \varphi \in D\left(A_{\mathbb{C}}\right), \quad \psi \in \mathbf{H}_{\mathbb{C}}
$$

We observe that formula $(10)$ can be also obtained by applying Corollary 8 with $f(\xi)=\xi$ and $g(\xi)=\operatorname{Re} \xi$ to relation (9). At that for $\omega \subset \mathbb{R}$ we have $g^{-1}(\omega)=\omega+i \mathbb{R} \subset \mathbb{C}$ and this is why the operators $E^{A_{\mathbb{C}}}(\omega)$ satisfy $(7)$. By Corollary 10, the operators $E^{A_{\mathbb{C}}}(\omega)$ commute with the operator $J$ and therefore, are the complexifications of some operators $E^{A_{\mathbb{R}}}(\omega)$ and $E^{A_{\mathbb{R}}}\left(\sigma^{\operatorname{Re}}\left(N_{\mathbb{R}}\right)\right)=\mathbf{1}_{\mathbb{R}}$.

It follows from (4) that the operator $B_{\mathbb{C}}$ satisfies the representation

$$
\left\langle B_{\mathbb{C}} \varphi, \psi\right\rangle_{\mathbb{C}}=\int_{\mathbb{C}} i \operatorname{Im} \xi d E_{\varphi \psi}^{N_{\mathbb{C}}}(\xi), \quad \varphi \in D\left(B_{\mathbb{C}}\right), \quad \psi \in \mathbf{H}_{\mathbb{C}} .
$$

We employ Corollary 8 with $f(\xi)=\xi$ and $g(\xi)=\operatorname{Im} \xi$ and as a result, we obtain:

$$
\left\langle B_{\mathbb{C}} \varphi, \psi\right\rangle_{\mathbb{C}}=\int_{-\infty}^{+\infty} i \beta d E_{\varphi \psi}^{B_{\mathbb{C}}}(\beta),
$$

where $E^{B_{\mathbb{C}}}(\omega)=E^{N_{\mathbb{C}}}(\mathbb{R}+i \omega)$. By the transformations we get

$$
\begin{aligned}
\left\langle B_{\mathbb{C}} \varphi, \psi\right\rangle_{\mathbb{C}} & =\int_{0}^{+\infty} \beta d i E_{\varphi \psi}^{B_{\mathbb{C}}}(\beta)+\int_{-\infty}^{0} \beta d i E_{\varphi \psi}^{B_{\mathbb{C}}}(\beta) \\
& =\int_{0}^{+\infty} \beta d i E_{\varphi \psi}^{B_{\mathbb{C}}}(\beta)+\int_{0}^{+\infty}-\beta d i E_{\varphi \psi}^{B_{\mathbb{C}}}(-\beta) \\
& =\int_{0}^{+\infty} \beta d\left(i E_{\varphi \psi}^{B_{\mathbb{C}}}(\beta)-i E_{\varphi \psi}^{B_{\mathbb{C}}}(-\beta)\right)=\int_{0}^{+\infty} \beta d G_{\varphi \psi}^{B_{\mathbb{C}}}(\beta),
\end{aligned}
$$

where $G^{B_{\mathbb{C}}}(\omega)=i E^{B_{\mathbb{C}}}(\omega)-i E^{B_{\mathbb{C}}}(-\omega)=i E^{N_{\mathbb{C}}}(\mathbb{R}+i \omega)-i E^{N_{\mathbb{C}}}(\mathbb{R}-i \omega)$. It is easy to check that the operators $G^{B_{\mathbb{C}}}(\omega)$ commute with the operator $J$ and therefore, they are the complexifications of some operators $G^{B_{\mathbb{R}}}(\omega)$. 
The operator $P_{\mathbb{C}}=E^{N_{\mathbb{C}}}\left(\sigma^{0}\left(N_{\mathbb{R}}\right)\right)$ is a self-adjoint projector, commutes with the operator $J$ by Proposition 9 and is the complexification of some self-adjoint projector in $P_{\mathbb{R}}$. The statement that the operators $G^{B_{\mathbb{C}}}(\omega), \omega \in \Sigma$, is the complex skew resolution of the identity in $\mathcal{R}\left(\mathbf{1}_{\mathbb{R}}-P_{\mathbb{R}}\right)$ supported on $\sigma^{\operatorname{Im}}+\left(N_{\mathbb{R}}\right) \subset \mathbb{R}^{+}$can be checked straightforwardly.

Since all the operators of the family $E^{N_{\mathbb{C}}}(\omega), \omega \in \Sigma$, commute, it follows from formulae (7) and (8) that each two operators $E^{A_{\mathbb{C}}}(\omega)$ and $G^{B_{\mathbb{C}}}\left(\omega^{\prime}\right)$ commute as well. This is why the operators $E^{A_{\mathbb{R}}}(\omega)$ and $G^{B_{\mathbb{R}}}\left(\omega^{\prime}\right)$ commute, too. The proof is complete.

\section{Second spectral theorem (on the Complex Plane)}

Spectral representation (5) in Theorem 15 can not be used for constructing a complete functional calculus. Because of this we provide one more spectral theorem allowing us to formulate the theorem on functional calculus. In this section we also employ the notion of the real resolution of the identity and the real skew resolution of the identity in the subspace $\mathcal{R}\left(\mathbf{1}_{\mathbb{R}}-P_{\mathbb{R}}\right)$, but as the sets $\Omega$ and $\Delta$, we mean Borel sets in the upper complex half-plane $\mathbb{C}^{+}$.

Theorem 16. Let $N_{\mathbb{R}}: D\left(N_{\mathbb{R}}\right) \subset \mathbf{H}_{\mathbb{R}} \rightarrow \mathbf{H}_{\mathbb{R}}$ be a normal operator. Then there exist a selfadjoint projector $P_{\mathbb{R}}$, a real resolution of the identity $E^{+N_{\mathbb{R}}}$ and a real skew resolution of the identity $G^{+N_{\mathbb{R}}}$ in the subspace $\mathcal{R}\left(\mathbf{1}_{\mathbb{R}}-P_{\mathbb{R}}\right)$ supported on $\sigma^{+}\left(N_{\mathbb{R}}\right)$, for which the relation

$$
\left\langle N_{\mathbb{R}} \varphi, \psi\right\rangle_{\mathbb{R}}=\int_{\mathbb{C}^{+}} \operatorname{Re} \xi d E_{\varphi \psi}^{+N_{\mathbb{R}}}(\xi)+\int_{\mathbb{C}^{+}} \operatorname{Im} \xi d G_{\varphi \psi}^{+N_{\mathbb{R}}}(\xi), \quad \varphi \in D\left(N_{\mathbb{R}}\right), \quad \psi \in \mathbf{H}_{\mathbb{R}}
$$

At that, each two operators $E^{+N_{\mathbb{R}}}(\omega)$ and $G^{+N_{\mathbb{R}}}\left(\omega^{\prime}\right)$ commute.

The complexification $P_{\mathbb{C}}$ of the projector $P_{\mathbb{R}}$ and the complexifications $E^{+N_{\mathbb{C}}}(\omega)$ and $G^{+N_{\mathbb{C}}}(\omega)$ of the operators $E^{+N_{\mathrm{R}}}(\omega)$ and $G^{+N_{\mathrm{R}}}(\omega)$ are related with the spectral decomposition $E^{N_{\mathrm{C}}}$ of the complexification $N_{\mathbb{C}}$ of the operator $N_{\mathbb{R}}$ by the formulae

$$
\begin{aligned}
P_{\mathbb{C}} & =E^{N_{\mathbb{C}}}\left(\sigma^{0}\left(N_{\mathbb{R}}\right)\right), \\
E^{+N_{\mathbb{C}}}(\omega) & =E^{N_{\mathbb{C}}}(\omega)+E^{N_{\mathbb{C}}}(\bar{\omega})-E^{N_{\mathbb{C}}}(\omega \cap \bar{\omega})=E^{N_{\mathbb{C}}}(\omega \cup \bar{\omega}), \\
G^{+N_{\mathbb{C}}}(\omega) & =i\left(E^{N_{\mathbb{C}}}(\omega)-E^{N_{\mathbb{C}}}(\bar{\omega})\right) .
\end{aligned}
$$

Proof. By Proposition 13, we represent the complexification $N_{\mathbb{C}}$ of the operator $N_{\mathbb{R}}$ as $N_{\mathbb{C}}=A_{\mathbb{C}}+B_{\mathbb{C}}$, where $A_{\mathbb{C}}$ is a self-adjoint operator and $B_{\mathbb{C}}$ is skew-adjoint. We consider representation (3) of the operator $A_{\mathbb{C}}$ and apply Corollary 8 with $f(\xi)=\operatorname{Re} \xi, g(\xi)=\operatorname{Re} \xi+i|\operatorname{Im} \xi|$. As a result we obtain

$$
A_{\mathbb{C}}=\int_{\mathbb{C}} \operatorname{Re} \xi d E^{N_{\mathbb{C}}}(\xi)=\int_{\mathbb{C}^{+}} \operatorname{Re} \xi d E^{+N_{\mathbb{C}}}(\xi) .
$$

Since $g^{-1}(\omega)=\omega \cup \bar{\omega}$ for $\omega \subset \mathbb{C}^{+}$, the operators $E^{+N_{\mathbb{C}}}(\omega)$ are determined by formula (13). After the transformations of representation (4) of the operator $B_{\mathbb{C}}$ we have

$$
B_{\mathbb{C}}=\int_{\mathbb{C}} i \operatorname{Im} \xi d E^{N_{\mathbb{C}}}(\xi)=\int_{\mathbb{C}^{+}} i \operatorname{Im} \xi d E^{N_{\mathbb{C}}}(\xi)-\int_{\mathbb{C}^{+}} i \operatorname{Im} \xi d E^{N_{\mathbb{C}}}(\bar{\xi})=\int_{\mathbb{C}^{+}} \operatorname{Im} \xi d G^{+N_{\mathbb{C}}}(\xi),
$$

where the operators $G^{+N_{\mathbb{C}}}(\omega)$ are given by formula $(14)$. Thus,

$$
N_{\mathbb{C}}=A_{\mathbb{C}}+B_{\mathbb{C}}=\int_{\mathbb{C}^{+}} \operatorname{Re} \xi d E^{+N_{\mathbb{C}}}(\xi)+\int_{\mathbb{C}^{+}} \operatorname{Im} \xi d G^{+N_{\mathbb{C}}}(\xi) .
$$

It is easy to check that the operators $E^{+N_{\mathbb{C}}}(\omega)$ and $G^{+N_{\mathbb{C}}}(\omega)$ commute with the operator $J$.

As $P_{\mathbb{R}}$ we take the restriction of the self-adjoint projector $E^{N_{\mathbb{C}}}\left(\sigma^{0}\left(N_{\mathbb{R}}\right)\right)$ on $\mathbf{H}_{\mathbb{R}}$. As $E^{+N_{\mathbb{R}}}(\omega)$ and $G^{+N_{\mathbb{R}}}(\omega)$ we choose the restrictions of $E^{+N_{\mathbb{C}}}(\omega)$ and $G^{+N_{\mathbb{C}}}(\omega)$ on $\mathbf{H}_{\mathbb{R}}$. The statement that the families $E^{+N_{\mathrm{R}}}(\omega), \omega \in \Sigma$, and $G^{+N_{\mathrm{R}}}(\omega), \omega \in \Sigma$, are a real resolution of the identity and a real skew resolution of the identity in $\mathcal{R}\left(\mathbf{1}_{\mathbb{R}}-P_{\mathbb{R}}\right)$ supported on $\sigma^{+}\left(N_{\mathbb{R}}\right)$ can be checked by straightforward calculations. At that, relation (11) is obviously true. Since all operators in 
the family $E^{N_{\mathbb{C}}}(\omega), \omega \in \Sigma$, commute, formulae 113 and (14) imply that each two operators $E^{+N_{\mathbb{C}}}(\omega)$ and $G^{+N_{\mathbb{C}}}\left(\omega^{\prime}\right)$ commute, too. This is why the operators $E^{+N_{\mathbb{R}}}(\omega)$ and $G^{+N_{\mathbb{R}}}\left(\omega^{\prime}\right)$. The proof is complete.

We shall call the families of the operators $E^{+N_{\mathbb{R}}}(\omega), \omega \in \Sigma$, and $G^{+N_{\mathbb{R}}}(\omega), \omega \in \Sigma$, the real spectral decomposition of a normal operator $N_{\mathbb{R}}$.

\section{ThEOREM ON FUNCTIONAL CALCULUS}

The real spectral decomposition constructed in Section 4 can be employed for determining the operator $\Psi_{\mathbb{R}}(f)$ acting in $\mathbf{H}_{\mathbb{R}}$. In the next theorem we show that the complexification of the operator $\Psi_{\mathbb{R}}(f)$ coincides with the operator $\Psi_{\mathbb{C}}(f)$ defined in Section 3 .

Theorem 17. Let $N_{\mathbb{R}}: D\left(N_{\mathbb{R}}\right) \subset \mathbf{H}_{\mathbb{R}} \rightarrow \mathbf{H}_{\mathbb{R}}$ be a normal operator and $f: \mathbb{C} \rightarrow \mathbb{C}$ be a Borel measurable function possessing the property $\overline{f(\xi)}=f(\bar{\xi}), \xi \in \mathbb{C}$. We define the operator $\Psi_{\mathbb{R}}(f): D\left(\Psi_{\mathbb{R}}(f)\right) \subset \mathbf{H}_{\mathbb{R}} \rightarrow \mathbf{H}_{\mathbb{R}}$ by the formula

$$
\Psi_{\mathbb{R}}(f)=\int_{\mathbb{C}^{+}} \operatorname{Re} f d E^{+N_{\mathrm{R}}}+\int_{\mathbb{C}^{+}} \operatorname{Im} f d G^{+N_{\mathrm{R}}}
$$

with the domain $D\left(\Psi_{\mathbb{R}}(f)\right)=\left\{\varphi \in \mathbf{H}_{\mathbb{R}}: \int_{\mathbb{C}^{+}}|f|^{2} d E_{\varphi \varphi}^{+N_{\mathbb{R}}}<\infty\right\}$. Then the complexification of the operator $\Psi_{\mathbb{R}}(f)$ coincides with the operator $\Psi_{\mathbb{C}}(f)$.

Proof. It is easy to check that the relation

$$
\Psi_{\mathbb{C}}(f)=\Psi_{\mathbb{C}}(\operatorname{Re} f)+\Psi_{\mathbb{C}}(i \operatorname{Im} f)
$$

holds true, where in view of the property $f(\bar{\xi})=\overline{f(\xi)}$

$$
\begin{aligned}
\Psi_{\mathbb{C}}(\operatorname{Re} f) & =\int_{\mathbb{C}} \operatorname{Re} f(\xi) d E^{N_{\mathbb{C}}}(\xi)=\int_{\mathbb{C}^{+}} \operatorname{Re} f(\xi) d E^{+N_{\mathbb{C}}}(\xi), \\
D\left(\Psi_{\mathbb{C}}(\operatorname{Re} f)\right) & =\left\{\varphi \in \mathbf{H}_{\mathbb{C}}: \int_{\mathbb{C}}(\operatorname{Re} f(\xi))^{2} d E_{\varphi \varphi}^{N_{\mathbb{C}}}(\xi)<\infty\right\} \\
& =\left\{\varphi \in \mathbf{H}_{\mathbb{C}}: \int_{\mathbb{C}^{+}}(\operatorname{Re} f(\xi))^{2} d E_{\varphi \varphi}^{+N_{\mathbb{C}}}(\xi)<\infty\right\}, \\
\Psi_{\mathbb{C}}(i \operatorname{Im} f) & =\int_{\mathbb{C}} i \operatorname{Im} f(\xi) d E^{N_{\mathbb{C}}}(\xi)=\int_{\mathbb{C}^{+}} \operatorname{Im} f(\xi) d G^{+N_{\mathbb{C}}}(\xi), \\
D\left(\Psi_{\mathbb{C}}(\operatorname{Re} f)\right) & =\left\{\varphi \in \mathbf{H}_{\mathbb{C}}: \int_{\mathbb{C}}(\operatorname{Im} f(\xi))^{2} d E_{\varphi \varphi}^{N_{\mathbb{C}}}(\xi)<\infty\right\} \\
& =\left\{\varphi \in \mathbf{H}_{\mathbb{C}}: \int_{\mathbb{C}^{+}}(\operatorname{Im} f(\xi))^{2} d E_{\varphi \varphi}^{+N_{\mathbb{C}}}(\xi)<\infty\right\} .
\end{aligned}
$$

It is obvious that the operators $\Psi_{\mathbb{C}}(\operatorname{Re} f)$ and $\Psi_{\mathbb{C}}(i \operatorname{Im} f)$ are the complexifications of the operators $\int_{\mathbb{C}^{+}} \operatorname{Re} f d E^{+N_{\mathrm{R}}}$ and $\int_{\mathrm{C}^{+}} \operatorname{Im} f d G^{+N_{\mathrm{R}}}$, respectively. The proof is complete.

We formulate the properties of the obtained real functional calculus.

Theorem 18. Let $E^{+N_{\mathbb{R}}}(\omega), G^{+N_{\mathbb{R}}}(\omega), \omega \in \Sigma$, be the spectral decomposition of a normal operator $N_{\mathbb{R}}: D\left(N_{\mathbb{R}}\right) \subset \mathbf{H}_{\mathbb{R}} \rightarrow \mathbf{H}_{\mathbb{R}}$ and $f: \mathbb{C} \rightarrow \mathbb{C}$ be a Borel measurable function possessing the property $\overline{f(\xi)}=f(\bar{\xi})$. Then the operator $\Psi_{\mathbb{R}}(f)$ possesses the properties:

(a) For each $\varphi \in D\left(\Psi_{\mathbb{R}}(f)\right)$ the identity

$$
\left\|\Psi_{\mathbb{R}}(f) \varphi\right\|_{\mathbb{R}}^{2}=\int_{\mathbb{C}^{+}}|f|^{2} d E_{\varphi \varphi}^{+N_{\mathbb{R}}}
$$

holds. 
(b) The operator $\Psi_{\mathbb{R}}(f)$ is normal and the relation holds:

$$
\Psi_{\mathbb{R}}(f)^{*}=\Psi_{\mathbb{R}}(\bar{f})=\int_{\mathbb{C}^{+}} \operatorname{Re} f d E^{+N_{\mathbb{R}}}-\int_{\mathbb{C}^{+}} \operatorname{Im} f d G^{+N_{\mathbb{R}}}
$$

(c) The spectral decomposition of the adjoint operator $N_{\mathbb{R}}^{*}$ is related with the spectral decomposition of the operator $N_{\mathbb{R}}$ by the relations

$$
E^{+N_{\mathbb{R}}^{*}}(\omega)=E^{+N_{\mathbb{R}}}(\omega), \quad G^{+N_{\mathbb{R}}^{*}}(\omega)=\left(G^{+N_{\mathbb{R}}}(\omega)\right)^{*}=-G^{+N_{\mathbb{R}}}(\omega), \quad \omega \in \Sigma .
$$

(d) If the function $f$ is bounded, then the operator $\Psi_{\mathbb{R}}(f)$ is bounded.

(e) For all Borel measurable functions $f, g: \mathbb{C} \rightarrow \mathbb{C}$ obeying $\overline{f(\xi)}=f(\bar{\xi}), \overline{g(\xi)}=g(\bar{\xi})$, the inclusions

$$
\Psi_{\mathbb{R}}(f)+\Psi_{\mathbb{R}}(g) \subseteq \Psi_{\mathbb{R}}(f+g), \quad \Psi_{\mathbb{R}}(f) \Psi_{\mathbb{R}}(g) \subseteq \Psi_{\mathbb{R}}(f g)
$$

hold true. If at that the function $g$ is bounded then

$$
\Psi_{\mathbb{R}}(f)+\Psi_{\mathbb{R}}(g)=\Psi_{\mathbb{R}}(f+g), \quad \Psi_{\mathbb{R}}(f) \Psi_{\mathbb{R}}(g)=\Psi_{\mathbb{R}}(f g) .
$$

Proof. (a) Let $\varphi \in D\left(\Psi_{\mathbb{R}}(f)\right)$. We have

$$
\left\|\Psi_{\mathbb{R}}(f) \varphi\right\|_{\mathbb{R}}^{2}=\left\|\Psi_{\mathbb{C}}(f) \varphi\right\|_{\mathbb{C}}^{2}=\int_{\mathbb{C}}|f|^{2} d E_{\varphi \varphi}^{N_{\mathbb{C}}}=\int_{\mathbb{C}^{+}}|f|^{2} d E_{\varphi \varphi}^{+N_{\mathbb{R}}}
$$

(b) It is obvious that the operator $\Psi_{\mathbb{C}}(\bar{f})$ is the complexification of the operator $\Psi_{\mathbb{R}}(\bar{f})$. It follows from Theorem $6(\mathrm{~b})$ that $\Psi_{\mathbb{C}}(f)^{*}=\Psi_{\mathbb{C}}(\bar{f})$. This is why $\Psi_{\mathbb{R}}(f)^{*}=\Psi_{\mathbb{R}}(\bar{f})$.

(c) This property is implied by (b).

Properties (d), (e) are obvious. The proof is complete.

Example 1. We consider the Cauchy problem for the homogeneous equation [22]

$$
\dot{x}(t)=N_{\mathbb{R}} x(t), \quad x(0)=b,
$$

where $N_{\mathbb{R}}: D\left(N_{\mathbb{R}}\right) \subset \mathbf{H}_{\mathbb{R}} \rightarrow \mathbf{H}_{\mathbb{R}}$ is a normal operator, whose spectrum is located in the left complex half-plane. We introduce the notation $\exp _{t}(\xi)=e^{\xi t}$. One can show that the generalized solution [22] of problem (15) satisfies the representation

$$
\begin{aligned}
x(t) & =\Psi_{\mathbb{R}}\left(\exp _{t}\right) b=\int_{\mathbb{C}^{+}} \operatorname{Re} \exp _{t}(\xi) d E^{+N_{\mathbb{R}}}(\xi) b+\int_{\mathbb{C}^{+}} \operatorname{Im} \exp _{t}(\xi) d G^{+N_{\mathbb{R}}}(\xi) b \\
& =\int_{\{\alpha+i \beta: \alpha \in \mathbb{R}, \beta \geqslant 0\}} e^{\alpha t} \cos \beta t d E^{+N_{\mathbb{R}}}(\alpha+i \beta) b+\int_{\{\alpha+i \beta: \alpha \in \mathbb{R}, \beta \geqslant 0\}} e^{\alpha t} \sin \beta t d G^{+N_{\mathbb{R}}}(\alpha+i \beta) b .
\end{aligned}
$$

We stress that both terms in the right hand side belong to $\mathbf{H}_{\mathbb{R}}$.

Example 2. In the Hilbert space $\mathbf{H}_{\mathbb{R}}=L_{2}[0,2 \pi]$ with the scalar product

$$
\left\langle z_{1}, z_{2}\right\rangle_{\mathbb{R}}=\frac{1}{2 \pi} \int_{0}^{2 \pi} z_{1}(s) z_{2}(s) d s
$$

we consider the operator

$$
N_{\mathbb{R}}=\frac{d^{2}}{d s^{2}}+a_{1} \frac{d}{d s}+a_{2},
$$

where $a_{1}, a_{2} \in \mathbb{R}$, with the domain formed by the functions $z \in W_{2}^{2}[0,2 \pi]$ satisfying the periodic boundary conditions

$$
z(0)=z(2 \pi), \quad z^{\prime}(0)=z^{\prime}(2 \pi) .
$$

By straightforward calculations we check that the eigenfunctions of the complexification $N_{\mathbb{C}}$ of the operator $N_{\mathbb{R}}$ are of the form: $\psi_{k}(s)=e^{i k s}, k \in \mathbb{Z}$, and

$$
\sigma\left(N_{\mathbb{R}}\right)=\sigma\left(N_{\mathbb{C}}\right)=\left\{\xi_{k}=-k^{2}+i a_{1} k+a_{2}: k \in \mathbb{Z}\right\} .
$$

It is obvious that $\bar{\xi}_{k}=\xi_{-k}$ and $\overline{\psi_{k}(\cdot)}=\psi_{-k}(\cdot)$. 
For a Borel measurable function $f: \mathbb{C} \rightarrow \mathbb{C}$ with the property $\overline{f(\xi)}=f(\bar{\xi})$ we have

$$
\begin{aligned}
\left(\Psi_{\mathbb{C}}(f) z\right)(s) & =\sum_{k=-\infty}^{+\infty} f\left(\xi_{k}\right)\left\langle z, \psi_{k}\right\rangle_{\mathbb{C}} \psi_{k}(s)=\sum_{k=-\infty}^{+\infty}\left\langle z(\cdot), \overline{f\left(\xi_{k}\right)} \psi_{k}(\cdot) \overline{\psi_{k}(s)}\right\rangle_{\mathbb{C}} \\
& =f\left(a_{2}\right)\left\langle z(\cdot), \psi_{0}(\cdot)\right\rangle_{\mathbb{C}}+\sum_{k=1}^{+\infty}\left\langle z(\cdot), 2 \operatorname{Re}\left\{f\left(\xi_{k}\right) \overline{\psi_{k}(\cdot)} \psi_{k}(s)\right\}\right\rangle_{\mathbb{C}} d \xi
\end{aligned}
$$

We observe that

$$
\begin{aligned}
\operatorname{Re}\left\{f\left(\xi_{k}\right) \overline{\psi_{k}(\cdot)} \psi_{k}(s)\right\}= & \operatorname{Re} f\left(\xi_{k}\right) \operatorname{Re}\left\{\psi_{k}(\cdot)\right\} \operatorname{Re}\left\{\psi_{k}(s)\right\}+\operatorname{Re} f\left(\xi_{k}\right) \operatorname{Im}\left\{\psi_{k}(\cdot)\right\} \operatorname{Im}\left\{\psi_{k}(s)\right\} \\
& +\operatorname{Im} f\left(\xi_{k}\right) \operatorname{Re}\left\{\psi_{k}(\cdot)\right\} \operatorname{Im}\left\{\psi_{k}(s)\right\}-\operatorname{Im} f\left(\xi_{k}\right) \operatorname{Im}\left\{\psi_{k}(\cdot)\right\} \operatorname{Re}\left\{\psi_{k}(s)\right\}
\end{aligned}
$$

This is why for $z \in \mathbf{H}_{\mathbb{R}}$ we have

$$
\Psi_{\mathbb{R}}(f) z=f\left(a_{2}\right) E^{+N_{\mathbb{R}}}\left(\left\{a_{2}\right\}\right) z+\sum_{k=1}^{+\infty} \operatorname{Re} f\left(\xi_{k}\right) E^{+N_{\mathrm{R}}}\left(\left\{\xi_{k}\right\}\right) z+\sum_{k=1}^{+\infty} \operatorname{Im} f\left(\xi_{k}\right) E^{+G_{\mathrm{R}}}\left(\left\{\xi_{k}\right\}\right) z
$$

where

$$
\begin{aligned}
& \left(E^{+N_{\mathrm{R}}}\left(\left\{a_{2}\right\}\right) z\right)(s)=\frac{1}{2 \pi} \int_{0}^{2 \pi} z(y) d y, \\
& \left(E^{+N_{\mathrm{R}}}\left(\left\{\xi_{k}\right\}\right) z\right)(s)=\frac{\cos k s}{\pi} \int_{0}^{2 \pi} z(y) \cos k y d y+\frac{\sin k s}{\pi} \int_{0}^{2 \pi} z(y) \sin k y d y, \\
& \left(E^{+G_{\mathrm{R}}}\left(\left\{\xi_{k}\right\}\right) z\right)(s)=\frac{\sin k s}{\pi} \int_{0}^{2 \pi} z(y) \cos k y d y-\frac{\cos k s}{\pi} \int_{0}^{2 \pi} z(y) \sin k y d y .
\end{aligned}
$$

In particular, for the function $\exp _{t}(\xi)=e^{\xi t}$ we have

$$
\begin{aligned}
\Psi_{\mathbb{R}}\left(\exp _{t}\right)= & e^{a_{2} t} E^{+N_{\mathbb{R}}}\left(\left\{a_{2}\right\}\right)+\sum_{k=1}^{+\infty} e^{\left(-k^{2}+a_{2}\right) t} \cos \left(a_{1} k t\right) E^{+N_{\mathbb{R}}}\left(\left\{\xi_{k}\right\}\right) \\
& +\sum_{k=1}^{+\infty} e^{\left(-k^{2}+a_{2}\right) t} \sin \left(a_{1} k t\right) E^{+G_{\mathbb{R}}}\left(\left\{\xi_{k}\right\}\right) .
\end{aligned}
$$

Thus, in order to calculate a function of the operator $N_{\mathbb{R}}$ including the operator exponent with the parameter $t$, there is no need to pass to the complexification.

\section{BIBLIOGRAPHY}

1. K. Maurin. Methods of Hilbert space. Mir, Moscow (1965). [Metody przestrzeni Hilberta, Pánstwowe Wydawnictvo Naukowe, Warsawa (1959) (in Polish); Methods of Hilbert space, PWNPolish Scientific Publishers, Warszawa (1967).]

2. M.A. Najmark. Normed rings. Nauka, Moscow (1968). [Wolters-Noordhoff Publ. Groningen (1970).]

3. W. Rudin. Functional analysis. [ McGraw-Hill Series in Higher Mathematics. McGraw-Hill Book Comp., New York (1973).]

4. E. Hille, R.S. Phillips. Functional analysis and semigroups. Colloquium Publications. 31. Amer. Math. Soc., Providence, R. I. (1957).

5. N. Dunford, J.T. Schwartz. Linear operators. Part II: Spectral theory. Self-adjoint operators in Hilbert space. Pure Appl. Math. 7. Interscience Publishers, New York (1963).

6. G. Golub, Ch.F. Van Loan. Matrix computations. The Johns Hopkins Univ. Press. Baltimore (1996).

7. J.W. Demmel. Applied numerical linear algebra. SIAM, Philadelphia (1997).

8. A.C. Antoulas. Approximation of large-scale dynamical systems. SIAM, Philadelphia (2005). 
9. I.P. Gavrilyuk, V.L. Makarov. Exponentially convergent algorithms for the operator exponential with applications to inhomogeneous problems in Banach spaces // SIAM J. Numer. Anal. 43:5, 2144-2171 (2005).

10. L. Lopez, V. Simoncini. Analysis of projection methods for rational function approximation to the matrix exponential // SIAM J. Numer. Anal. 44:2, 613-635 (2006).

11. V.G. Kurbatov, M.N. Oreshina. On finding approximate solution to a linear second order differential equation // Vestnik VGU. Ser. fizika, matematika. 2, 173-188 (2003). (in Russian).

12. V.G. Kurbatov, I.V. Kurbatova. Krylov subspace methods of approximate solving differential equations from the point of view of functional calculus // Eurasian Math. J. 3:4, 53-80 (2012).

13. V.G. Kurbatov, M.N. Oreshina. Interconnect macromodelling and approximation of matrix exponent // Analog Integrated Circuits and Signal Processing. 40:1, 5-19 (2004).

14. M.N. Oreshina. Approximate solution of a parabolic equation with the use of a rational approximation to the operator exponential // Differ. Uravn. 407-417 (2017). [Diff. Equat. 53:3, 398-408 (2017).]

15. L.V. Kantorovich, G.P. Akilov. Functional analysis. Pergamon Press, Oxford (1982).

16. A.G. Baskakov, A.S. Zagorskii. Spectral theory of linear relations on real Banach spaces // Matem. Zametki. 81:1, 17-31 (2007). [Math. Notes. 81:1, 15-27 (2007).]

17. I.M. Glazman, Yu.I. Lyubich. Finite-dimensional linear analysis: A systematic presentation in problem form. Nauka, Moscow (1969). [The M.I.T. Press, Cambridge. (1974).]

18. A.V. Pechkurov. Complexification of ordered pair of linear operators // Vestnik VGU. Ser. fizika, matematika. 2, 143-147 (2007). (in Russian).

19. M.N. Oreshina. Spectral theorem for self-adjoint operators in real Hilbert space // Vestnik VGU. Ser. fizika, matematika. 3, 120-133 (2015). (in Russian).

20. P.R. Halmos. Measure theory. University Series in Higher Mathematics. D. Van Nostrand Co., Inc. New York (1950).

21. N. Bourbaki. Integration. Measures on locally compact spaces. Continuation of measure. Integration of measure. Measures on separable spaces. Nauka, Moscow (1977). (in Russian).

22. S.G. Krein. Linear differential equations in Banach space. Nauka, Moscow (1967). (in Russian).

Maria Nikolaevna Oreshina,

Lipetsk State Technical University,

Moskovskaya str. 30,

398600, Lipetsk, Russia

E-mail: m_oreshina@mail.ru 